\title{
MULTIPLICATIVE LIE HIGHER DERIVATIONS OF UNITAL ALGEBRAS WITH IDEMPOTENTS
}

\author{
DONG HAN AND FENG WEI
}

Abstract. Let $\mathscr{R}$ be a commutative ring with identity and $\mathscr{A}$ be a unital algebra with nontrivial idempotent $e$ over $\mathscr{R}$. Motivated by Benkovič's systematic and powerful work [2, 3, 4, 5, 6, 7, 8], we will study multiplicative Lie higher derivations (i.e. those Lie higher derivations without additivity assumption) on $\mathscr{A}$ in this article. Let $D=\left\{L_{k}\right\}_{k \in \mathbb{N}}$ be a multiplicative Lie higher derivation on $\mathscr{A}$. It is shown that under suitable assumptions, $D=\left\{L_{k}\right\}_{k \in \mathbb{N}}$ is of standard form; i.e. each component $L_{k}(k \geqslant 1)$ can be expressed through an additive higher derivation and a central mapping vanishing on all commutators of $\mathscr{A}$.

Mathematics subject classification (2010): 15A78, 15A86, 16W10.

Keywords and phrases: Lie higher derivation, higher derivation, additive derivation, unital algebra, generalized matrix algebra.

\section{REFERENCES}

[1] K. ARdakov And K. A. BRown, Ring-theoretic properties of Iwasawa algebras: a survey, Doc. Math., (2006), Extra Volume, 7-33.

[2] D. BenKovič, Lie derivations on triangular matrices, Linear Multilinear Algebra, 55 (2007), 619626.

[3] D. Benkovič, Generalized Lie derivations on triangular algebras, Linear Algebra Appl., 434 (2011), $1532-1544$.

[4] D. Benkovič, Lie triple derivations on triangular matrices, Algebra Colloq., 18 (2011), Special Issue No. 1, 819-826.

[5] D. Benkovič, Lie triple derivations of unital algebras with idempotents, Linear Multilinear Algebra, 63 (2015), 141-165.

[6] D. BENKOVIČ AND D. EREMITA, Multiplicative Lie $n$-derivations of triangular rings, Linear Algebra Appl., 436 (2012), 4223-4240.

[7] D. BENKOVIČ AND M. GRAŠIČ, Generalized derivations on unital algebras determined by action on zero products, Linear Algebra Appl., 445 (2014), 347-368.

[8] D. BENKOVIČ AND N. ŠIROVNIK, Jordan derivations of unital algebras with idempotents, Linear Algebra Appl., 437 (2012), 2271-2284.

[9] L. CHEN AND J.-H. ZHANG, Nonlinear Lie derivations on upper triangular matrices, Linear Multilinear Algebra, 56 (2008), 725-730.

[10] Z.-X. CHEN AND Z.-K. XIAO, Nonlinear Lie triple derivations on parabolic subalgebras of finitedimensional simple Lie algebras, Linear Multilinear Algebra, 60 (2012), 645-656.

[11] W.-S. Cheung, Lie derivations of triangular algebras, Linear Multilinear Algebra, 51 (2003), 299 310 .

[12] J. Cuntz, Simple $C^{*}$-algebras generated by isometries, Commun. math. Phys., 57 (1977), $173-185$.

[13] J. Cuntz And W. KRIEGER, A class of $C^{*}$-algebras and topological Markov chains, Invent. Math., 56 (1980), 251-268.

[14] Y.-Q. DU AND Y. WANG, Lie derivations of generalized matrix algebras, Linear Algebra Appl., 437 (2012), 2719-2726.

[15] M. Ferrero And C. Haetinger, Higher derivations and a theorem by Herstein, Quaest. Math., 25 (2002), 249-257. 
[16] M. Ferrero And C. Haetinger, Higher derivations of semiprime rings, Comm. Algebra, 30 (2002), 2321-2333.

[17] A. FoŠnER, F. WEI AND Z.-K. XIAO, Nonlinear Lie-type derivations of von Neumann algebras and related topics, Colloq. Math., 132 (2013), 53-71.

[18] K. E. GeHLEs, Properties of Cherednik algebras and graded Hecke algebras, Ph. D. Thesis, University of Glasgow, 2006.

[19] P. Goldstein, On graph $C^{*}$-algebras, J. Aust. Math. Soc., 72 (2002), 153-160.

[20] D. HAN, Lie-type higher derivations on operator algebras, Bull. Iran Math. Soc., 40 (2014), 1169 1194.

[21] H. Hasse And F. K. SChmidt, Noch eine Begründung der Theorie der höheren Differentialquotienten in einem algebraischen Funktionenkörper einer Unbestimmten, (German), J. Reine Angew. Math., 177 (1937), 215-237.

[22] N. Heerema, Higher derivations and automorphisms of complete local rings, Bull. Amer. Math. Soc., 76 (1970), 1212-1225.

[23] P. N. Jewell, Continuity of module and higher derivations, Pacific J. Math., 68 (1977), 91-98.

[24] P.-S. Ji, R.-R. LiU AND Y.-Z. ZHAO, Nonlinear Lie triple derivations of triangular algebras, Linear Multilinear Algebra, 60 (2012), 1155-1164.

[25] R. V. KAdison AND J. R. Ringrose, Fundamentals of the theory of operator algebras, Vol. I-II. Academic Press, San Diego, 1983-1986.

[26] S. KöNIG AND C.-C. XI, A characteristic free approach to Brauer algebras, Trans. Amer. Math. Soc., 353 (2000), 1489-1505.

[27] P. A. KRYLOV, Isomorphism of generalized matrix rings, Algebra and Logic, 47 (2008), 258-262.

[28] J. Li AND J. GuO, Characterizations of higher derivations and Jordan higher derivations on CSL algebras, Bull. Aust. Math. Soc., 83 (2011), 486-499.

[29] R. J. LoY, Continuity of higher derivations, Proc. Amer. Math. Soc., 37 (1973), 505-510.

[30] F. Lu, Lie triple derivations on nest algebras, Math. Nachr., 280 (2007), 882-887.

[31] C. R. Miers, Lie derivations of von Neumann algebras, Duke Math. J., 40 (1973), 403-409.

[32] C. R. Miers, Lie triple derivations of von Neumann algebras, Proc. Amer. Math. Soc., 71 (1978), $57-61$.

[33] J. B. Miller, Homomorphisms, higher derivations and derivations on associative algebras, Acta Sci. Math. (Sezeged), 28 (1967), 221-231.

[34] J. B. MiLLeR, Higher derivations on Banach algebras, Amer. J. Math., 92 (1970), 301-331.

[35] X.-F. QI, Characterization of Lie higher derivations on triangular algebras, Acta Math. Sinica, 29 (2013), 1007-1018.

[36] X.-F. QI AND J.-C. Hou, Lie higher derivations on nest algebras, Commun. Math. Res., 26 (2010), $131-143$.

[37] S.-L. SUN AND X.-F. MA, Lie triple derivations of nest algebras on Banach spaces, Linear Algebra Appl., 436 (2012), 3443-3462.

[38] Y. WANG, Lie n-derivations of unital algebras with idempotents, Linear Algebra Appl., 458 (2014), $512-525$.

[39] Y. WANG AND Y. WANG, Multiplicative Lie $n$-derivations of generalized matrix algebras, Linear and Multilinear Algebra, 438 (2013), 2599-2616.

[40] F. WEI AND Z.-K. XIAO, Higher derivations of triangular algebras and its applications, Linear Algebra Appl., 435 (2011), 1034-1054.

[41] Z.-K. XIAO AND F. WEI, Jordan higher derivations on triangular algebras, Linear Algebra Appl., 432 (2010), 2615-2622.

[42] Z.-K. XIAO AND F. WeI, Nonlinear Lie higher derivations on triangular algebras, Linear and Multilinear Algebra, 60 (2012), 979-994.

[43] Z.-K. XIAO AND F. WeI, Lie triple derivations of triangular algebras, Linear Algebra Appl., 437 (2012), 1234-1249.

[44] W.-Y. YU AND J.-H. ZhANG, Nonlinear Lie derivations of triangular algebras, Linear Algebra Appl., 432 (2010), 2953-2960.

[45] J.-H. ZHANG, B.-W. WU AND H.-X. CAO, Lie triple derivations of nest algebras, Linear Algebra Appl., 416 (2006), 559-567.

[46] X. Zhang, R.-L. An AND J.-C. Hou, Characterization of Higher derivations on CSL algebras, Expo. Math., 31 (2013), 392-404. 
Operators and Matrices www.ele-math.com oamele-math.com 